\title{
Formulación estratégica. Caso: empresas del sector alimentos y bebidas del estado Carabobo
}

\author{
Strategic Formulation. Case: Business Sector State Food \\ and Beverages Carabobo
}

\author{
Ana Emilia Cordero Borjas ${ }^{1}$
}

Recibido: 31 de enero, 2011

Aceptado: 21 de febrero, 2011

\section{Resumen}

El objetivo de esta investigación es analizar el proceso de formulación estratégica de un grupo de empresas del sector alimentos y bebidas del Estado Carabobo, Venezuela. Para ello se llevó a cabo un estudio no experimental, con especial énfasis en un diseño transeccional descriptivo, y se aplicó un instrumento a una muestra no probabilística de once organizaciones dedicadas a la fabricación de productos alimenticios y bebidas en el Estado Carabobo. La conclusión a que se llegó es que en el grupo de empresas encuestadas no hay una clara tendencia o identificación con respecto de alguna de las escuelas del pensamiento estratégico, en particular, en lo referido al proceso de formulación estratégica; en otras palabras no existe suficiente homogeneidad en las respuestas que permitan detectar qué escuela del pensamiento estratégic tiene mayor presencia entre las empresas. Al considerar el carácter prismático del proceso de formulación estratégica, se puede afirmar que las escuelas con mayor influencia al momento de hacer la formulación estratégica son de Planificación, de Posicionamiento, Cultural, Ambiental y de Configuración.

Palabras clave: Planificación estratégica, formulación estratégica, pensamiento estratégico.

\begin{abstract}
The purpose of this paper is to analyze the strategic process of a group of companies in the food and beverage sector of Carabobo State, Venezuela. To achieve it a non-experimental study was conducted with special emphasis on transectional descriptive design and an instrument was applied to a non probabilistic sample taken from eleven organizations of the food and beverage manufacturing industry in the Carabobo State, Venezuela. We concluded that in the group there is no clear trend or identification with respect to some of the schools of strategic thought, in particular, as regards to the strategic process; that is to say, there is not enough uniformity in the responses to identify which school of strategic thought (from the application of its premises) has a greater presence in some of the companies. Considering the prismatic nature of the strategic process, it can be said that the schools with more influence at the time of making the strategic formulation are Planning, Positioning, Cultural, Environmental and Configuration.
\end{abstract}

Keywords: Strategic planning, strategy formulation, strategic thought.

1. Profesora investigadora de la Escuela de Administración Comercial y Contaduría Pública de la Universidad de Carabobo, Venezuela. Postgrado en Gerencia de Recursos Humanos (Facultad de Ciencias Económicas y Sociales de la Universidad de Carabobo.<aecorder@gmail.com>. 


\section{INTRODUCCIÓN}

El estudio de la planificación estratégica como campo académico en mucho más reciente que su práctica real. Los estudios sistematizados datan de la década de los años 60 del siglo pasado, mientras que su aplicación se remonta a siglos antes de Cristo. Los constantes cambios en el entorno organizacional han llevado a diversos investigadores a focalizar algunos de sus estudios en la planificación de estrategias a fin de analizar la interacción de la organización con su entorno y la definición de cursos de acción que garanticen el éxito organizacional.

El pensamiento estratégico como perspectiva integradora de la organización y su entorno, que combina métodos analíticos y elasticidad mental en los procesos de formulación, implantación, ejecución y control de la administración estratégica, visualizando cómo será la organización en el futuro, es objeto de análisis en algunas investigaciones, entre las cuales destaca la realizada recientemente por Ramos (2009). Este investigador estudió un grupo de empresas con sede en el Valle de Aburrá (Colombia), provenientes de diversos sectores económicos y sin distinción de su antigüedad. En este estudio se concluye que, considerando el tiempo de fundación de las empresas, en ellas se hacen perceptibles los cambios generados por procesos tales como la globalización, la competitividad, la innovación de los modelos de negocio, por citar algunos. En las empresas de menos de una década de existencia se aprecia que primero se define la estrategia, mientras que en las empresas de más de 10 años de funcionamiento, con marcado acento en las sociedades de más de 20 años, sucede todo lo contrario, es decir, la estructura condiciona la estrategia.

De Weffer (2009) estudia 63 empresas inscritas en la cámara de industriales del estado Zulia a fin de analizar el proceso de formulación de estrategias en empresas medianas y grandes ubicadas en manufactura, distribución eléctrica y minería. En este estudio se evidenció la inexistencia de sistemas de planificación rigurosa, a excepción de la empresa de generación y distribución eléctrica del estado, ya que el resto se basa en la experiencia de sus dueños y las pautas de empresa internacionales.

Piñeiro, Quintero y Faria (2007) determinaron la relación que existe entre el pensamiento estratégico y la eficiencia de la gerencia pública en las corporaciones locales de la Región Zuliana, tomando como muestra 110 directores que laboran en las alcaldías y paramunicipales de la Costa Oriental del Lago. Los investigadores concluyen que existe un nivel de asociación bajo, lo cual indica que la actitud, la cultura y las herramientas empleadas dentro de las alcaldías y paramunicipales no son determinantes para alcanzar una eficiencia dentro de las mismas. La variable pensamiento estratégico se maneja mediante el desarrollo de diversos factores tanto internos como externos.

Por su parte, Perozo (2006) realizó una investigación descriptiva y de campo teniendo como objeto de estudio 42 gerentes de diversas empresas de servicios del sector petrolero del estado Zulia, Venezuela. Las conclusiones del estudio demostraron que las empresas seleccionadas se ubicaron en: a) una categoría moderadamente eficiente en relación con la presencia del pensamiento estratégico; b) en cuanto a la caracterización de los procesos para la gestión del talento humano se determinó moderadamente eficiente; y c) la relación entre el pensamiento estratégico y gestión del talento humano en los gerentes corporativos es casi nula. Por ello se establece como conclusión que las variables estudiadas se comportan en forma independiente una de la otra.

Silvestri, Silvestri y Hernández (2006), al determinar la relación que existe entre el pensamiento estratégico y el éxito gerencial de los directivos de las organizaciones empresariales en el sector químico de la costa Oriental y Occidental del Lago, concluyen que existe una alta relación entre las variables de estudio. Aquí se logra determinar que tanto la actitud como los procesos, las herramientas y la cualidad son factores claves para lograr un éxito gerencia; orientado al logro de los objetivos organizacionales y de sus miembros.

Giacinti (2003), en una investigación menos reciente, pretende incentivar en el sector mundial de agronegocios 
de la manzana la reflexión sobre la necesidad de una mayor toma de decisiones por parte de los productores y los gerentes, basada en la realidad agronómica y comercial de cada región productiva, para lograr la optimización económica de la misma. El autor señala que existen «factores "no naturales" como la evolución de la paridad real de las monedas frente al dólar estadounidense, que tienen un alto impacto económico en el sector en los últimos años» (50).

Uno de los hallazgos más importantes de la investigación de Giacinti (2003) es la siguiente:

La inexistencia de grupos homogéneos importantes de pensamiento estratégico en el negocio de manzanas a nivel mundial, a excepción de algunos pares de países de pensamiento similar, como Estados Unidos y Australia, Nueva Zelanda y Dinamarca, Alemania y España, así como los técnicos argentinos entre sí, tanto de las empresas como los independientes (60).

Ante ello, el autor reflexiona sobre la necesidad de una mayor afinidad en el pensamiento estratégico entre productores y técnicos, lo cual es indispensable para comenzar el cambio de actitud que permita establecer el camino hacia un modelo "exitoso".

Las investigaciones reseñadas no solo evidencian el interés por analizar las diversas variables que intervienen en la planificación estratégica, la formulación estratégica y el pensamiento estratégico, sino que generan inquietudes en torno a ello. Es decir, no se puede precisar una clara tendencia en cuanto a la formulación y pensamiento estratégico en particular. Lo que sí se puede reconocer es que en la medida que se le asocien a otras variables como antigüedad de la organización, gestión de talentos humanos, actividad económica o éxito empresarial, entre otras variables, la relación varía significativamente.

Es importante destacar que la presente investigación se centra en el sector alimentos y bebidas del Estado Carabobo (Venezuela) por su relevancia en la economía venezolana. Según el estudio de Mercado, Córdova y Testa (2007), la agroindustria en Venezuela en los últimos treinta años ha respondido entre un " 11 y $12 \%$ del producto industrial bruto (PIB) no petrolero; de este aporte, $55 \%$ ha correspondido a productos agropecuarios y $45 \%$ a manufactura de alimentos y bebidas" (98). Dentro de las industrias transformadoras, la industria de alimentos ocupa un lugar importante, pues es superada con márgenes ajustados por las industrias química, petroquímica y farmacéutica (Mercado, Córdova \& Testa, 2007)

Estos mismos autores afirman que la industria agroalimentaria a nivel mundial se halla en el ojo del huracán por sus implicaciones sociales y económicas:

\begin{abstract}
A pesar de no ser considerada dentro de las actividades más determinantes de la competitividad global, continua mereciendo tratamiento especial por parte de la mayoría de las naciones, entre otras cosas, porque es vital para la sobrevivencia de la población y constituye el eje de la economía de muchos países, en especial los más pobres (86).
\end{abstract}

Como sostienen Castillo y Morales (2006), en los países desarrollados el sector agroindustrial durante los últimos años se ha dedicado a la sustitución de productos estandarizados por productos con características diversas que ofrezcan a los consumidores una gama mucho más amplia de posibilidades. Estas variedades no habrían podido lograrse con un aparato agroindustrial rígido, que no se adaptara a los innovaciones que estaban ocurriendo. Estos cambios, según los autores, se fundamentan en "la flexibilidad productiva, la cual ha permitido a la industria agroalimentaria iniciar un proceso de transformación tendente a configurar formas técnico organizativas" (59).

Se debe mencionar que las modificaciones en la industria y la distribución han sido impulsadas gracias a la aplicación de avances tecnológicos, en especial de la informática en el sector de los servicios. Dichos avances han promovido la evolución de las formas organizativas y de los procesos de automatización de la producción industrial. Castillo y Morales afirman que

Los mismos han permitido que este sistema de relaciones correspondientes a la producción, circulación y venta de alimentos pueda responder rápida- 
mente a un mercado que está en permanente cambio y determinar, en última instancia, la eficiencia de toda la red productiva cuando ésta tenga que dirimir sus posibilidades en la arena de la competencia (59).

Una característica importante de la agroindustria en general es que su crecimiento es poco flexible, ya que se ve condicionado por la población mundial, originando que la competencia por los mercados de consumidores sea muy intensa. Las empresas de alimentos y bebidas, especialmente en el mundo desarrollado, intentan aumentar su rentabilidad y la mejora de su desempeño a través de estrategias para explotar intensivamente cada aspecto del negocio que ofrezca visos de competitividad (Mercado, Córdova \& Testa, 2007).

En Venezuela, al igual que en gran parte del mundo, se han dado procesos de concentración de la agroindustria y resurgimiento de espacios de producción local. Dicha situación configura la industria agroalimentaria venezolana en dos segmentos claramente diferenciados, según afirman los autores arriba mencionados. Tales segmentos son los siguientes:

a. Una fracción mayoritaria de la producción que corre por cuenta de filiales de multinacionales y algunos grupos nacionales. Este segmento emplea modernas técnicas de producción y se adecuan a estándares internacionales de calidad y control de procesos. Las empresas están ubicadas en las regiones Capital, Central y Centro-occidental, donde hay grandes mercados de consumo.

b. Pequeñas empresas, muchas de carácter cooperativo, como producto del resurgimiento de espacios de producción local en una amplia franja de la geografía nacional. En este caso, se emplean procesos productivos tradicionales que pueden calificarse en la categoría de tecnologías apropiadas.

Aproximadamente $60 \%$ de las grandes organizaciones dedicadas a la fabricación de productos alimenticios y bebidas en el país están concentradas en los estados Aragua, Carabobo (20.77\%) y Miranda (BCV, INE). Este sector genera una cantidad significativa de empleos directos e indirectos, sustentando así no solo su importancia económica, sino social en estas regiones de alta concentración demográfica. Debido a su importancia, y a fin de describir la realidad actual del sector alimentos y bebidas a nivel nacional, es indispensable remitirse al más reciente informe de la Cámara Venezolana de la Industria de Alimentos (CAVIDEA), el cual refleja la perspectiva que tienen sus afiliados sobre la situación actual de sus organizaciones durante el I trimestre del 2010. El informe es producto de la consulta a 53 afiliados de CAVIDEA, del cual se desprende lo siguiente:

a. 75\% afirmó que la situación actual del sector alimentos se ha comportado de manera desfavorable.

b. Las principales causas que originan que la situación actual de ese $75 \%$ sea desfavorable son las siguientes: controles gubernamentales (24\%); falta de materia prima (20\%); exceso de permisología ${ }^{1}(19 \%)$; falla de energía (18\%); nuevas leyes $(7 \%)$ y baja demanda (7\%), entre otros.

c. Los principales problemas que dificultaron el desempeño productivo de estas organizaciones para el I trimestre de 2010, fueron los siguientes: control de cambio (20\%); control de precios (20\%); problemas de materia prima (16\%); obtención de certificados de insuficiencia o no producción (14\%); créditos con proveedores en el exterior ( $8 \%$ ); fallas en el suministro de envases y empaques (7\%); competencia de productos importados (2\%); disponibilidad de recurso humano (2\%) e inseguridad (1\%).

d. $62 \%$ dice que el desempeño de las ventas y rentabilidad en la organización en el I trimestre de 2010 ha disminuido.

e. $91 \%$ afirma que el comportamiento del sector alimentos para el II trimestre de 2010 será regular y $6 \%$ afirma que será malo.

1 Según Alexis Márquez Rodríguez (2005), permisología es el "conjunto de permisos que se requieren, especialmente los que emanan de organismos oficiales, para una actividad determinada, como constituir empresas, realizar obras, prestar ciertos servicios, entre otros". [Documento en línea] 
La realidad de la agroindustria en general, y en especial la situación actual del sector manufacturero dedicado a la fabricación de alimentos y bebidas en Venezuela, conlleva a reflexionar sobre la necesidad del estudio del pensamiento estratégico (destacando en esta investigación el proceso de formulación estratégica) en este sector. El propósito es identificar las perspectivas de integración organización-entorno que estas empresas ponen en práctica actualmente a fin de adecuarse a los cambios que emergen de las características propias de un sector económico tradicionalmente complejo.

Por tanto, y a fin de contribuir a la reflexión, análisis, discusión y disertación sobre el tema, esta investigación examina el proceso de formulación estratégica de un grupo de empresas del sector dedicado a la fabricación de alimentos y bebidas del Estado Carabobo, Venezuela. Para ello, en primer lugar se analizarán los conceptos fundamentales vinculados al proceso de formulación estratégica. En segundo lugar se describirán las principales escuelas de la evolución del pensamiento estratégico. En tercer lugar se determinará el proceso de formulación estratégica de un grupo de empresas del sector alimentos y bebidas carabobeño a través de un trabajo de campo.

\section{FORMULACIÓN ESTRATÉGICA: EVOLUCIÓN DEL PENSAMIENTO ESTRATÉGICO}

Por lo ya expuesto, y a fin de alcanzar el propósito de esta investigación, es indispensable hacer una revisión de aquellos conceptos fundamentales vinculados al proceso de formulación estratégica.

\section{Estrategia: orígenes y vínculo con la administración}

Rumelt, Schendel y Teece (1994) sostienen que la gestión estratégica como campo académico en mucho más reciente que su aplicación real. Si bien la fecha de su concepción es algo incierta, la dirección estratégica dentro del campo académico es sin duda un niño de la década de 1960. En las organizaciones es común utilizar la palabra estrategia cuando se trata de definir el rumbo de las mismas, convirtiéndose en objeto de estudio de académicos, investigadores, gremios, entre otros grupos de interés vinculados al mundo organizacional. La búsqueda de la etimología de la palabra estrategia permite determinar que su procedencia se deriva de la unión de dos palabras de origen griego: stratos, que significa ejército, y agein, que significa conducir, guiar. Como el ambiente militar es el contexto en el cual se encuentran las raíces de esta palabra, la actividad del estratega consistía en proyectar, ordenar y dirigir las operaciones militares para conseguir el triunfo.

Von Neumann y Morgenstern (1948) en su teoría de juegos, iniciaron el uso de la estrategia en el mundo de los negocios. Esta teoría proporciona una visión unificada para todas las clases de situaciones conflictivas sin tener en cuenta si su origen es la guerra, la política o el negocio. A fin de analizar la estrategia como aspecto fundamental de la administración actual, es importante considerar a los diversos autores que desde este campo la han definido. Entre los autores se encuentra Newman (1951), quien afirma que la estrategia es una serie de actos no aislados que ejecuta una empresa, que son seleccionados de acuerdo con una situación concreta.

En 1962, en su obra Strategy and Structure, Chandler definió estrategia empresarial a partir del análisis de cuatro grandes organizaciones estadounidenses de principios del siglo XX: Sears Roebuck, Standar Oil of New Jersey, DuPont y General Motor. Chandler definió la estrategia como el elemento que determinaba las metas básicas de una empresa, a largo plazo, así como la adopción de cursos de acción y la asignación de los recursos necesarios para alcanzar estas metas.

Posteriormente, Ansoff (1965) considera que la estrategia es el lazo común entre las actividades de la organización y las relaciones producto-mercado, que definen la naturaleza esencial de los negocios en que la organización está involucrada y los negocios que planea para el futuro. El autor identifica cuatro componentes de la estrategia: 1) ámbito producto-mercado, 2) un vector crecimiento, 3) ventajas competitivas, y 4) la sinergia vista como la aptitud de una empresa para triunfar en 
una nueva actividad. Por su parte, Peter Woods (1985) señala que las estrategias son formas de llevar a cabo metas; son conjuntos de acciones identificables, orientadas a fines más amplios y generales.

Rumelt (1974) se refiere a la estrategia desde el punto de vista del direccionamiento de la organización; es decir, a partir de la relación entre la definición de los objetivos y la selección de los productos o servicios. También se debe tener en cuenta las políticas sobre el posicionamiento de la empresa en relación con su entorno y el nivel apropiado de enfoque y diversidad. Adicionalmente considera el diseño de la estructura y de los sistemas administrativos que se emplean para establecer el engranaje de actividades.

Por su parte, ante el proceso de formulación estratégica, Drucker (1994) asevera que se requieren gerentes que analicen la situación presente y que la cambien en caso de ser necesario, lo que implica que se debe tener una visión de los recursos con que se cuenta y de los que se deberían tener. Para Daft (2004), la estrategia es el «plan de acción que establece la asignación de recursos y otras actividades para enfrentar el ambiente y ayudar a obtener las metas de la organización» (243). Thompson y Strickland (2004) afirman que la estrategia de una empresa consiste en los esfuerzos competitivos y los enfoques de negocio que los administradores utilizan para satisfacer a los clientes, competir exitosamente y alcanzar los objetivos de la organización (10).

Si se consideran cada una de las definiciones de estrategia presentadas, es importante reconocer que existen tantos enunciados como autores se consulten. Sin embargo, un elemento común entre todas ellas es que la estrategia determina el o los cursos de acción para alcanzar las metas u objetivos planteados en la organización. Otro elemento que goza de reconocimiento entre la mayoría de los investigadores es que la definición de la estrategia, tal como planteó Chandler, guarda una estrecha relación con la definición de la estructura organizacional. Así lo estima Daft (2004) cuando dice que «La estrategia está vinculada a la estructura y a la cultura conforme la empresa cambia su manera fundamental de hacer negocios y permite que las iniciativas fluyan de abajo arriba y viceversa, y surja de los nexos con los clientes y aliados» (375).

Luego de haber precisado los aspectos comunes en las diversas definiciones de estrategia revisadas, es importante mencionar la investigación desarrollada por García y Sabater (2004) quienes indican que existen muchas clasificaciones sobre tipos de estrategia, pero pocos trabajos que analicen las distintas clasificaciones estratégicas y que intenten encontrar las similitudes y coincidencias entre ellas, para así plantear una clasificación global en la que se incluye el mayor número de puntos coincidentes entre ellas. Por tanto, ambos autores se plantean encontrar una clasificación global, para lo cual seleccionaron y analizaron 21 clasificaciones de estrategias. Esta selección de clasificaciones repercutió en trabajos de investigación posteriores. Las clasificaciones estudiadas, según las citan García y Sabater (2004), son las siguientes: Ansoff (1965), Rumelt (1974), Buzzell, Gale y Sultan (1975), Hofer y Schendel (1978), Miles y Snow (1978), Miller y Friesen (1978), Vesper (1979), Abell (1980) Wissema Van Der Pol y Messer (1980), Porter (1982), Galbraith y Schendel (1983), Herbert y Deresky (1987), Chrisman, Hofer y Boulton (1988), Mintzberg (1988), Simmond (1990), Menguzzato y Renau (1991), Morrison y Roth (1992), Mundet y Paturel (1995), Bueno (1996), Wright, Kroll y Parnell (1998) y Beal (2000) (ver Tabla 1).

De la investigación realizada por García y Sabater (2004), ambos derivan la siguiente conclusión:

Existe una relación entre el momento del ciclo de vida en el que se encuentra la empresa y tipo de estrategia más implantado en esa etapa. Por las características de la empresa en cada una de las etapas, la empresa se plantea unas determinadas opciones estratégicas, tanto a nivel corporativo como de unidad de negocio y no otras. La estrategia a nivel de unidad de negocio, en las primeras etapas, sería una estrategia de diferenciación y más tarde la opción más frecuente sería una estrategia de costes. A nivel corporativo en la etapa de crecimiento la estrategia más elegida será una estrategia de expansión o diversificación frente a otras alternativas. 
Tabla 1.

Distintos Niveles de Definición de Estrategias

\begin{tabular}{lll}
\hline \multicolumn{1}{c}{ Grupo 1 } & \multicolumn{1}{c}{ Grupo 2 } & \multicolumn{1}{c}{ Grupo 3} \\
\hline $\begin{array}{c}\text { Solo contemplan tipos de estrategia a } \\
\text { nivel de unicidad de negocio }\end{array}$ & $\begin{array}{c}\text { Contemplan tipos de estrategia a nivel de } \\
\text { negocio más estrategias a nivel corporativo }\end{array}$ & $\begin{array}{c}\text { Contemplan las dos variables anteriores } \\
\text { más formas de llevarlas a cabo }\end{array}$ \\
\hline - Miles y Snow (1978) & - Ansoff (1965) & ・ Vesper (1979) \\
- Porter (1982) & - Rumelt (1974) & - Simmond (1990) \\
- Abell (1980) & - Buzzell et al. (1975) & - Morrison y Roth (1992) \\
- Chrisman, et al. (1988) & - Hofer y Schendel (1978) & Mundet y Paturel (1995) \\
- Wright et al. (1998) & - Miller y Friesen (1978) & \\
- Beal (2000) & Bueno (1996) \\
& - Galssema, et al. (1980) & \\
\hline
\end{tabular}

Fuente: García y Sabater (2004).

Hasta el momento hemos revisado algunas definiciones de estrategia con el objeto de precisar los aspectos en los cuales coinciden los diversos autores a lo largo de más de 50 años con respecto a ello. También hemos constatado, a través de la investigación de García y Sabater (2004), que existen distintos niveles de definición de la estrategia a partir de las variables que considere el estratega.

\section{Planificación Estratégica}

En esta sección se mencionan algunos conceptos sobre planificación estratégica para luego posteriormente hacer un análisis del proceso de formulación estratégica y del pensamiento estratégico. La planificación estratégica tiene su impulso en dos obras de las década de los 60 del siglo pasado: Política empresaria: textos y casos de Learned, Christensen, Andrews, y Guth (1965), y Estrategias Corporativas de Harry Igor Ansoff. La primera obra propone la articulación eficaz del potencial del cual dispone la empresa, reflejada en sus fuerzas y debilidades dentro de un medio ambiente que presenta amenazas limitantes. Pero, al mismo tiempo toma en cuenta las oportunidades de desarrollo, ello, a través de la utilización del modelo LCAG creado por estos autores en la Harvard Business School, modelo ampliamente conocido como DOFA o FODA.
Estrategias Corporativas, obra escrita por Harry Igor Ansoff, fue publicado en 1965. Aquí el autor le da más impulso a lo propuesto por Learned, Christensen, Andrews, y Guth. Si bien es cierto que el libro de Ansoff es contemporáneo con el de estos autores, no es sino en la década de los setenta que cobra popularidad. En su libro, Ansoff analiza la formulación del plan estratégico a partir del planteamiento y confrontación de los objetivos de la empresa, así como del análisis interno y externo.

La planificación estratégica es vista como un proceso en el cual se toma en cuenta el porvenir, las decisiones actuales, la definición de metas, objetivos, políticas y estrategias para desarrollar planes a mediano y largo plazo. Esto es lo que permite ver a futuro y adelantarse a los posibles problemas. Este método es conocido como Planeación a Largo Plazo, y su éxito se deriva de la atención continua de las cambiantes condiciones internas y externas de la organización, además de la formulación y ejecución de estrategias acorde a esas condiciones (Sallenave, 1993).

Serna (1999) afirma que la planeación estratégica es el proceso administrativo que permite desarrollar y mantener una relación viable entre los objetivos, los recursos de la organización y las cambiantes oportunidades 
del mercado. El objetivo de la planeación estratégica es modelar y remodelar los negocios y productos de la empresa, de manera que se combinen para producir un desarrollo y utilidades satisfactorios. Por su parte, Rosales (2001) considera que la planeación estratégica es un proceso en el cual las organizaciones desarrollan instrucciones y actividades que buscan determinar metas y objetivos a través del análisis interno y externo, adoptando cursos de acción específicos y asignando recursos para hacer posible la obtención de las metas de la organización.

La planificación estratégica, más allá de ser una herramienta para el directivo, es un proceso interactivo de arriba hacia abajo y de abajo hacia arriba en la organización, proceso en el cual la dirección general establece con frecuencia metas para la empresa apoyada en la información recibida de mercados y/o de las unidades inferiores. Estas, a su vez, determinan planes y presupuestos que son consolidados y corregidos por las unidades superiores, que vuelven a enviarlos hacia abajo, donde son nuevamente revisados hasta establecer la planificación definitiva. Según Daft, «la importancia del proceso de recolección y procesamiento de la información necesaria para consolidar la planificación se redimensiona especialmente en el mundo de la globalización, de la desregulación, de los avances tecnológicos y de la demografía y estilos de vida en cambio constante» (238).

Ronda (2004), en su estudio de 27 modelos de dirección estratégica aplicados entre los años 1984 al 2001, demuestra que la dirección estratégica consta de cuatro fases principales: (a) formulación, (b) implantación, (c) ejecución, y (d) control. Asimismo, se determinó que la fase a la cual se le ha brindado mayor atención es la formulación. Las demás fases, es decir la implantación, la ejecución y el control, aunque se mencionan, presentan insuficiencias que afectan la ejecución efectiva. Ronda también indica algunas regularidades en la dirección estratégica, entre las cuales destacan:

a. La interacción de la organización con su entorno, donde el efecto de los factores externos sobre la organización aunque sean del mismo sector de mercado, es único; es decir, lo que para una organización constituye una amenaza, para otra puede constituir una oportunidad.

b. Principios teórico-metodológicos, tales como la naturaleza holística del modelo de planificación estratégica, el carácter integrador y proactivo del mismo, la flexibilidad para adecuarse a las exigencias internas y externas, y la retroalimentación sistémica e interacción constante de todos los procesos de la planificación estratégica (incluyendo la fase de ejecución).

c. La necesaria correspondencia entre pensamiento, actitud e intención durante la formulación de la estrategia, donde es imprescindible el dominio de las herramientas y técnicas de la misma. Es necesaria la integración del nivel estratégico con el táctico y el operativo.

Para Ronda el «pensamiento estratégico es el conjunto de juicios, conceptos y habilidades conceptuales, técnicas y humanas necesarias para realizar, con éxito, el proceso de formulación, implantación, ejecución y control de la dirección estratégica» (p. 38). En tal sentido, Mintzberg ya en 1998 había establecido la diferencia entre la planificación estratégica y el pensamiento estratégico: a) la planeación estratégica se centra en el análisis y se relaciona con la articulación, elaboración y formalización de las estrategias existentes, y b) el pensamiento estratégico enfatiza la síntesis usando la intuición y la creatividad para crear una perspectiva integrada de la empresa.

Al considerar lo expuesto por Mintzberg (1998) y Ronda (2004), entre otros autores que han estudiado el pensamiento estratégico y con el propósito de analizar algunas de sus definiciones, en el siguiente apartado se presentan los aportes de algunos investigadores sobre el pensamiento estratégico y la relevancia del mismo en el proceso de formulación estratégica.

\section{Pensamiento Estratégico}

Partiendo de lo ya expuesto, en particular de la importancia de la correspondencia entre pensamiento, actitud 
e intención durante la formulación de la estrategia y de una visión holística de la organización y los factores que inciden sobre su desempeño, a continuación se presentan algunas definiciones sobre pensamiento estratégico:

a. Morrison (1994), analizando el artículo "La Caída y auge de Planificación Estratégica" de Mintzberg (1994). define el pensamiento estratégico como un trabajo de síntesis cuyo resultado representa una perspectiva integrada de la organización, no una visión muy precisa o formal. Para pensar estratégicamente se requiere de intuición, creatividad; establecen que las ideas pueden y deben surgir en cualquier momento, en cualquier lugar de la organización, generalmente, se hace mediante procesos desordenados de aprendizaje, no en reuniones formales de planificación.

b. Morrisey (1996) considera que el pensamiento estratégico individual incluye la aplicación del juicio basado en la experiencia para determinar las direcciones futuras, y el pensamiento estratégico de las empresas es la coordinación de mentes creativas dentro de una perspectiva que le permita a un negocio avanzar hacia el futuro de una manera satisfactoria para todos.

c. Ohmae (1998) define el pensamiento estratégico como la combinación de métodos analíticos y elasticidad mental utilizados para obtener ventajas competitivas.

d. Robert y Dias (2000) afirma que «el pensamiento estratégico es un proceso que ocurre en la mente del ejecutivo y le permite visualizar cómo será el aspecto de la organización en el futuro, y donde los integrantes de la misma se convierten en elementos claves» (58).

Por su parte, Cendrós (2001) formula la existencia de tres modalidades del pensamiento estratégico:

- Una primera modalidad es cuando se hace énfasis en la planificación. Esta abarca el proceso de formulación de estrategias, elaboración de la mi- sión de la empresa, detección de oportunidades y amenazas externas de la organización, definición de fortalezas y debilidades, establecimiento de objetivos a largo plazo, generación de estrategias alternativas y elección de estrategias concretas que se seguirán.

- La segunda modalidad del pensamiento estratégico, según Cendrós (2001), hace énfasis en la visión. En efecto, la visión es una declaración amplia y suficiente que provee el marco de referencia de lo que la empresa es y quiere ser en el futuro, señalando el rumbo, la dirección de la empresa y que sirviendo de guía en la formulación de estrategias y del reflejo de la misión, objetivos y estrategias de la organización (Serna, 1999).

- La tercera modalidad, y la más reciente para Cendrós, es la que hace énfasis en el aprendizaje de todos los miembros de la organización: promueve la difusión permanente de conocimientos, afianza la innovación y genera un ambiente de experimentación buscando nuevas formas de hacer las cosas.

Como afirman Huerta y De Bourg (2009), el pensamiento estratégico por su naturaleza lleva a una búsqueda de la comprensión entre la dialéctica entre el entorno y la organización. Estos programas pueden relacionar tanto las estrategias del entorno como las estrategias internas que tratan con la estructura de la organización y las actividades de control social.

De todo lo anterior, se puede afirmar que el pensamiento estratégico representa una perspectiva integradora de la organización y su entorno, que combina métodos analíticos y elasticidad mental en los procesos de formulación, implantación, ejecución y control de la administración estratégica a fin de obtener ventajas competitivas, visualizando cómo será el aspecto de la organización en el futuro. El pensamiento estratégico ha sido analizado muy especialmente por Mintzberg, por ello, seguidamente se hará una breve reseña de las escuelas del pensamiento estratégico propuestas por este investigador. 


\section{Escuelas del Pensamiento Estratégico}

Con el objetivo de hacer una breve descripción las principales escuelas de la evolución del pensamiento estratégico, se considerará la propuesta de Mintzberg quien establece diez escuelas, las cuales se pueden agrupar en dos conjuntos: (a) las escuelas prescriptivas, que se ocupan del modo como debería formularse la estrategia; y (b) las escuelas descriptivas, que consideran aspectos específicos de la creación de estrategias (ver Tabla 2)

Se han analizado algunos conceptos fundamentales vinculados al proceso de formulación estraté- gica, como son estrategia, planificación estratégica y pensamiento estratégico. Por su parte, también es importante la descripción de las principales escuelas de la evolución del pensamiento estratégico a fin de determinar el proceso de formulación estratégica de un grupo de empresas del sector químico del Estado Carabobo, tal como se planteó al inicio de esta investigación. A continuación se presentan en la Tabla 3 algunas premisas básicas de las escuelas del pensamiento estratégico mencionadas en la Tabla 2 y las cuales están estrechamente relacionadas con el proceso de formulación estratégica.

Tabla 2.

Escuelas del Pensamiento Estratégico

\begin{tabular}{lll}
\hline & Escuelas & La Estrategia Vista como un Proceso \\
\hline Planificación & De concepción \\
Diseño & Formal \\
Posicionamiento & Analítico \\
Empresarial & Visionario \\
Cognoscitiva & Mental \\
Aprendizaje & Emergente \\
& De poder & \\
Cultural & De negociación \\
Ambiental & Colectivo \\
& De configuración & Reactivo \\
& De transformación \\
\hline
\end{tabular}

Fuente: Adaptado de Mintzberg et al. (1999). 
Tabla 3.

Premisas Básicas de las Escuelas del Pensamiento Estratégico

\begin{tabular}{|c|c|}
\hline Escuela & Premisas \\
\hline \multirow{5}{*}{ Escuela de Diseño } & 1. La formulación de estrategia es un proceso deliberado \\
\hline & 2. La responsabilidad por el control y el conocimiento descansa en el directivo principal \\
\hline & 3. El modelo de formulación de estrategia es simple e informal \\
\hline & 4. Las mejores estrategias vienen de un proceso de diseño individualizado \\
\hline & $\begin{array}{l}\text { 5. El proceso de diseño está finalizado cuando las estrategias aparecen completamente formuladas } \\
\text { como parte de la visión }\end{array}$ \\
\hline \multirow{4}{*}{ Escuela de Planificación } & 1. Las estrategias provienen de un proceso controlado y consciente de planificación formal \\
\hline & $\begin{array}{l}\text { 2. El proceso de formulación de estrategias se desarrolla en etapas claras, cada una de ellas } \\
\text { delineada a través de listados y sustentada por técnicas }\end{array}$ \\
\hline & $\begin{array}{l}\text { 3. La responsabilidad por el proceso general de formulación de estrategias descansa en } \\
\text { el directivo superior }\end{array}$ \\
\hline & $\begin{array}{l}\text { 4. Luego de la formulación de estrategias se presta minuciosa atención a los objetivos, } \\
\text { presupuestos, programas y planes operativos de diversos tipos }\end{array}$ \\
\hline \multirow{5}{*}{$\begin{array}{l}\text { Escuela de } \\
\text { Posicionamiento }\end{array}$} & 1. Las estrategias son posiciones, específicamente comunes e identificables en el mercado \\
\hline & 2. El mercado (contexto) es económico y competitivo \\
\hline & $\begin{array}{l}\text { 3. El proceso de formulación de estrategia depende de la selección de estas posiciones en } \\
\text { base a un cálculo analítico }\end{array}$ \\
\hline & 4. Los analistas juegan un papel fundamental en este proceso \\
\hline & $\begin{array}{l}\text { 5. La estructura de mercado impulsa estrategias de posición premeditada que, a su vez, } \\
\text { impulsan la estructura organizacional }\end{array}$ \\
\hline
\end{tabular}

1. La estrategia existe en la mente del líder como una visión del futuro de la organización

2. El proceso de formulación de estrategia está arraigado en la experiencia y la intuición del líder

3. El líder promueve la visión manteniendo un estrecho control personal sobre la aplicación

Escuela Empresarial 4. La visión estratégica se premeditada en su visión general

5. La visión estratégica se vuelve flexible en la forma en que se despliegan los detalles de la visión

6. Es necesaria una estructura simple para que el líder visionario pueda contar con una considerable libertad de maniobra

1. La formulación de estrategia es un proceso que tiene lugar en la mente del estratega

Escuela Cognoscitiva $\quad$ 2. Las estrategias surgen como perspectivas (bajo la forma de conceptos, mapas, esquemas y marcos)

3. Las estrategias son difíciles de obtener

1. La creación de estrategia es un proceso de aprendizaje a lo largo del tiempo

2. En la formulación de estrategias, el sistema colectivo es el que aprende ya que en la organización existen muchos estrategas en potencia

Escuela de Aprendizaje 3. El papel del liderazgo es el de administrar el proceso de aprendizaje estratégico a través del cual pueden emerger las nuevas estrategias

4. Primero las estrategias aparecen como patrones salidos del pasado; solo después, posiblemente, como planes para el futuro; y por último, como perspectivas para orientar la conducta general 
1. La estrategia que puede resultar de este proceso tiende a tomar la forma de posiciones en el mercado

Escuela de Poder

2. La creación de estrategia es la interacción entre intereses locales y coaliciones cambiantes

3. La organización promueve su propio bienestar mediante el control o la cooperación con otras organizaciones

4. La organización promueve estrategias colectivas en diversas clases de cadenas y alianzas

1. La creación de estrategia es un proceso de interacción social

2. La cultura organizacional orientan la formulación de estrategias

Escuela Cultural $\quad 3$ La estrategia es premeditada

4. La cultura tiende a promover cambios de posición dentro de la perspectiva estratégica general de la organización

1. El entorno es el actor principal en el proceso de creación de estrategia

Escuela Ambiental

2. La organización puede responder a esas fuerzas del entorno

3. El liderazgo tiene como misión leer el entorno y asegurar una adaptación correcta por parte de la organización

1. La organización adopta una forma particular de estructura que combina con un tipo particular de contexto

2. La organización pasa eventualmente por algún proceso de transformación

Escuela de Configuración

3. La organización pasa por estados sucesivos de configuración y periodos de transformación

4. La clave de la gerencia estratégica de la organización está en reconocer la necesidad de transformación

5. El proceso de creación de estrategia deber ser realizado en el momento y contexto oportuno

Fuente: Adaptado de Mintzberg et al. (1999).

A partir de la revisión de los conceptos vinculados al proceso de formulación estratégica, donde destacan no solo la evolución de dichos conceptos y los puntos convergentes entre investigadores del tema, se puede afirmar que la formulación de estrategias en las organizaciones siempre ha sido un proceso de significativa importancia, siendo objeto de estudio dentro de la teoría de la administración por ser determinante del futuro y éxito de las organizaciones. A lo largo de los últimos 60 años, la formulación como proceso inicial de la planificación estratégica, ha tenido especial atención ya que no solo requiere de una perspectiva integradora de la organización y su entorno (pensamiento estratégico), sino que implica una conexión temporal de la organización al vincular su pasado con el presente y la situación futura deseada.

Todo lo anterior sirve de marco teórico referencial para este estudio que pretende analizar el proceso de formulación estratégica de un grupo de empresas del sector alimentos y bebidas del Estado Carabobo, Venezuela. Considerando este marco teórico referencial, en el siguiente apartado se detallará la metodología utilizada para el estudio descriptivo realizado en el sector alimentos y bebidas carabobeño.

\section{REFERENCIA EN EL SECTOR ALIMENTOS Y BEBIDAS DEL ESTADO CARABOBO}

\section{Metodología}

El estudio, según el nivel de investigación, es considerado descriptivo. Se recolectó y analizó información con el objetivo de determinar el proceso de formulación estratégica de un grupo de empresas del sector alimentos y bebidas del Estado Carabobo, Venezuela. Para alcanzar 
el objetivo propuesto se llevó a cabo un estudio no experimental, con especial énfasis en un diseño transeccional descriptivo.

Para ello se utilizó una encuesta como instrumento de recolección de información, aplicada a ejecutivos del área de recursos humanos o administración de cinco empresas: cuatro de ellas pertenecen al sector de fabricación de productos alimenticios y bebidas de la industria manufacturera del Estado Carabobo, Venezuela. La muestra utilizada es de carácter no probabilístico, de sujetos voluntarios y se tomó de acuerdo con la población determinada por el censo del Instituto Nacional de Estadística (INE) del 2005 que utiliza como base de datos CONINDUSTRIA. Aquí se especifica que este sector de actividad está conformado por 27 empresas de la Gran Industria. Para la selección se aplicó el siguiente criterio: en primer lugar pertenecer a la Gran Industria, y en segundo lugar a empresas de fabricación de productos alimenticios y bebidas de la industria manufacturera. De esa población se logró consultar a 11 empresas de capital privado, las cuales de manera voluntaria suministraron la información requerida. A continuación se hace una breve caracterización de la muestra consultada partiendo de la Clasificación Industrial Internacional Uniforme de todas las actividades económicas (CIIU) 4 (Tabla 4):
Sobre la base de las premisas de las escuelas del pensamiento estratégico (Tabla 3), se diseñó un cuestionario donde se formulan 43 afirmaciones sobre dichas premisas. Para ello se presentan afirmaciones de varias alternativas a través de una escala de medición tipo Lickert de cuatro (4) puntos, lo cual permitió aplicar una prueba estadística para el análisis descriptivo de dichas actividades objeto de estudio. La escala se diseñó de la siguiente manera:

\begin{tabular}{lc}
\hline \multicolumn{1}{c}{ Valoración cualitativa } & $\begin{array}{c}\text { Valoración } \\
\text { cuantitativa }\end{array}$ \\
\hline TA: Totalmente de acuerdo & 4 \\
MA: Moderadamente de acuerdo & 3 \\
MD: Moderadamente en desacuerdo & 2 \\
TD: Totalmente en desacuerdo & 1 \\
\hline
\end{tabular}

El cuestionario fue sometido al juicio de expertos para su validación y posteriormente se midió la confiabilidad del instrumento usando para ello el coeficiente de confiabilidad Alpha de Cronbach. Luego de calcular el coeficiente de confiabilidad, se puede afirmar que el instrumento diseñado es $96 \%$ confiable, considerando así la elevada estabilidad del mismo.

Tabla 4.

CIIU de Todas las Actividades Económicas 4

\begin{tabular}{|c|c|}
\hline Empresa $\mathrm{N}^{\circ}$ & CIIU 4 \\
\hline 1 & Elaboración de productos cárnicos * \\
\hline 2 & Elaboración de aceites y grasas de origen vegetal o animal * \\
\hline 3 & $\begin{array}{l}\text { Elaboración de aceites y grasas de origen vegetal o animal; molienda de otros } \\
\text { cereales; elaboración de cacao, chocolate y de productos de confitería * }\end{array}$ \\
\hline 4 & Elaboración de aceites y grasas de origen vegetal o animal * \\
\hline 5 & $\begin{array}{l}\text { Elaboración de aceites y grasas de origen vegetal o animal; elaboración de } \\
\text { productos cárnicos; elaboración de bebidas no alcohólicas } * * *\end{array}$ \\
\hline 6 & Elaboración de café $* *$ \\
\hline 7 & $\begin{array}{l}\text { Destilación, rectificación y mezcla de bebidas alcohólicas; producción de } \\
\text { alcohol etílico a partir de sustancias fermentadas } * *\end{array}$ \\
\hline 8 & Elaboración de frutas, legumbres y hortalizas $* * *$ \\
\hline 9 & Molienda de otros cereales * \\
\hline 10 & Elaboración de productos cárnicos * \\
\hline 11 & Elaboración de productos lácteos; elaboración de bebidas no alcohólicas ** \\
\hline
\end{tabular}

Fuente: elaboración propia (2010).

NOTA: * Elaboración de alimentos / ** Elaboración de bebidas / *** Elaboración de alimentos y bebidas 


\section{Resultados}

Con los datos recabados durante la aplicación del instrumento, se elaboró la Tabla 5, en la cual se reflejan los promedios obtenidos de las respuestas de las 11 empresas con relación a cada una de las preguntas y la variación en porcentaje. En ella se permite determinar una tendencia en cuanto al proceso de formulación estratégica de este grupo de empresas:

Tabla 5 .

Formulación Estratégica de un Grupo de Empresas del Sector Alimentos y Bebidas Carabobeño

\begin{tabular}{|c|c|c|}
\hline \multirow{2}{*}{ Premisas } & \multicolumn{2}{|c|}{ Medidas } \\
\hline & Promedio & Variación $(\%)$ \\
\hline 1. La formulación de estrategia es un proceso deliberado & 2.45 & 58.65 \\
\hline 2. La responsabilidad por el control y el conocimiento descansa en el directivo principal & 2.09 & 54.34 \\
\hline 3. El modelo de formulación de estrategia es simple e informal & 1.64 & 49.44 \\
\hline 4. Las mejores estrategias vienen de un proceso de diseño individualizado & 1.82 & 48.06 \\
\hline $\begin{array}{l}\text { 5. El proceso de diseño está finalizado cuando las estrategias aparecen completamente } \\
\text { formuladas como parte de la visión }\end{array}$ & 3.18 & 18.95 \\
\hline 6. Las estrategias provienen de un proceso controlado y consciente de planificación formal & 3.73 & 12.53 \\
\hline $\begin{array}{l}\text { 7. El proceso de formulación de estrategias se desarrolla en etapas claras, cada una de ellas } \\
\text { delineadas a través de listados y sustentada por técnicas }\end{array}$ & 3.82 & 10.59 \\
\hline $\begin{array}{l}\text { 8. La responsabilidad por el proceso general de formulación de estrategias descansa } \\
\text { en el directivo superior }\end{array}$ & 2.18 & 40.05 \\
\hline $\begin{array}{l}\text { 9. Luego de la formulación de estrategias, se presta minuciosa atención a los objetivos, } \\
\text { presupuestos, programas y planes operativos de diversos tipos }\end{array}$ & 3.82 & 10.59 \\
\hline 10. Las estrategias son posiciones, específicamente comunes e identificables en el mercado & 3.09 & 39.50 \\
\hline 11. El mercado (contexto) es económico y competitivo & 3.82 & 10.59 \\
\hline $\begin{array}{l}\text { 12. El proceso de formulación de estrategia depende de la selección de estas posiciones } \\
\text { sobre la base de un cálculo analítico }\end{array}$ & 3.55 & 19.39 \\
\hline 13. Los analistas juegan un papel fundamental en este proceso & 3.73 & 12.53 \\
\hline $\begin{array}{l}\text { 14. La estructura de mercado impulsa estrategias de posición premeditada que, } \\
\text { a su vez, impulsan la estructura organizacional }\end{array}$ & 3.64 & 18.54 \\
\hline 15. La estrategia existe en la mente del líder como una visión del futuro de la organización & 3.45 & 19.90 \\
\hline $\begin{array}{l}\text { 16. El proceso de formulación de estrategia está arraigado en la experiencia y la intuición } \\
\text { del líder }\end{array}$ & 2.91 & 39.06 \\
\hline 17. El líder promueve la visión manteniendo un estrecho control personal sobre la aplicación & 3.09 & 22.67 \\
\hline 18. La visión estratégica se premeditada en su visión general & 3.27 & 30.83 \\
\hline $\begin{array}{l}\text { 19. La visión estratégica se vuelve flexible en la forma en que se despliegan los detalles } \\
\text { de la visión }\end{array}$ & 3.55 & 14.73 \\
\hline $\begin{array}{l}\text { 20. Es necesaria una estructura simple para que el líder visionario pueda contar con } \\
\text { una considerable libertad de maniobra }\end{array}$ & 2.91 & 24.08 \\
\hline 21. La formulación de estrategia es un proceso que tiene lugar en la mente del estratega & 2.64 & 38.95 \\
\hline
\end{tabular}


22. Las estrategias surgen como perspectivas (bajo la forma de conceptos, mapas, esquemas y marcos)

3.36

1.64

3.18

3.64

3.36

2.64

45.75 posiblemente, como
la conducta general

28. La estrategia que puede resultar de este proceso tiende a tomar la forma de posiciones en el mercado

29. La creación de estrategia es la interacción entre intereses locales y coaliciones cambiantes

30. La organización promueve su propio bienestar mediante el control o la cooperación con otras organizaciones

31. La organización promueve estrategias colectivas en diversas clases de cadenas y alianzas

32. La creación de estrategia es un proceso de interacción social

33. La cultura organizacional orientan la formulación de estrategias

34. La estrategia es premeditada

35. La cultura tiende a promover cambios de posición dentro de la perspectiva estratégica general de la organización

36. El entorno es el actor principal en el proceso de creación de la estrategia

37. La organización puede responder a esas fuerzas del entorno

38. El liderazgo tiene como misión leer el entorno y asegurar una adaptación correcta por parte de la organización

39. La organización adopta una forma particular de estructura que combina con un tipo particular de contexto

40. La organización pasa eventualmente por algún proceso de transformación

41. La organización pasa por estados sucesivos de configuración y periodos de transformación

42. La clave de la gerencia estratégica de la organización está en reconocer la necesidad de transformación

43. El proceso de creación de estrategia deber ser realizado en el momento y contexto oportuno

Fuente: Elaboración propia (2010).

De acuerdo con los datos de la Tabla 5, se procedió a calcular el promedio ponderado y la variación promedio (\%) del grupo de afirmaciones vinculadas a cada escuela. Ello se hace con el objeto de identificar en primer lugar la escuela con mayor presencia al momento de formular estrategias dentro del grupo de empresas consultadas (Figura 1), y en segundo lugar para detectar la escuela cuya variación promedio sea menor (Figura 2). 


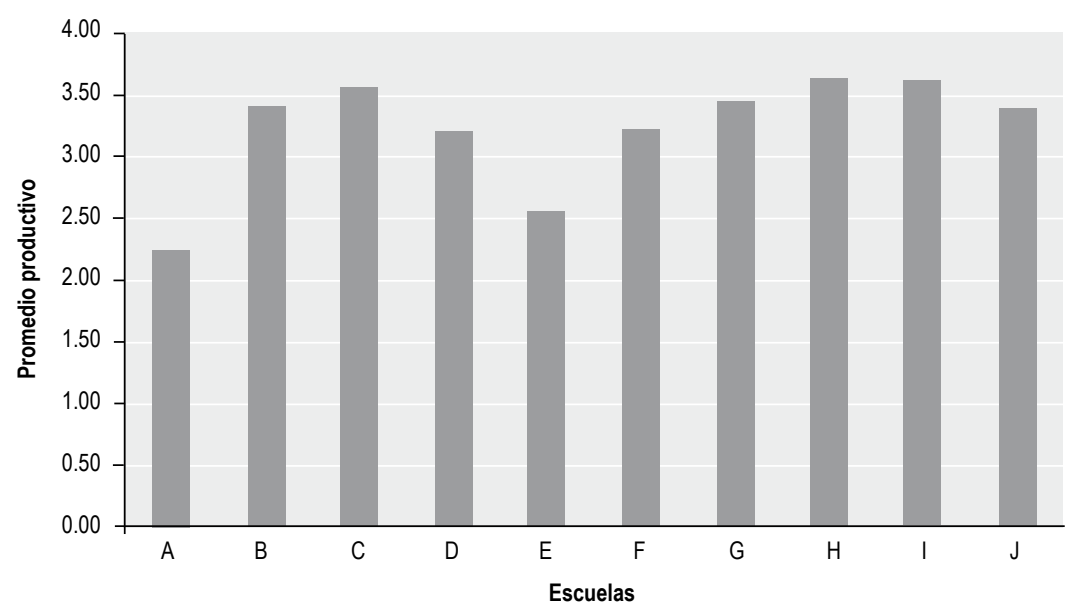

Figura 1. Escuelas prescriptivas: (A) Planificación; (B) Diseño; (C) Posicionamiento. Escuelas descriptivas: (D) Empresarial; (E) Cognoscitiva; (F) Aprendizaje; (G) De poder; (H) Cultural; (I) Ambiental; (J) De configuración.

Fuente: Elaboración propia (2010).

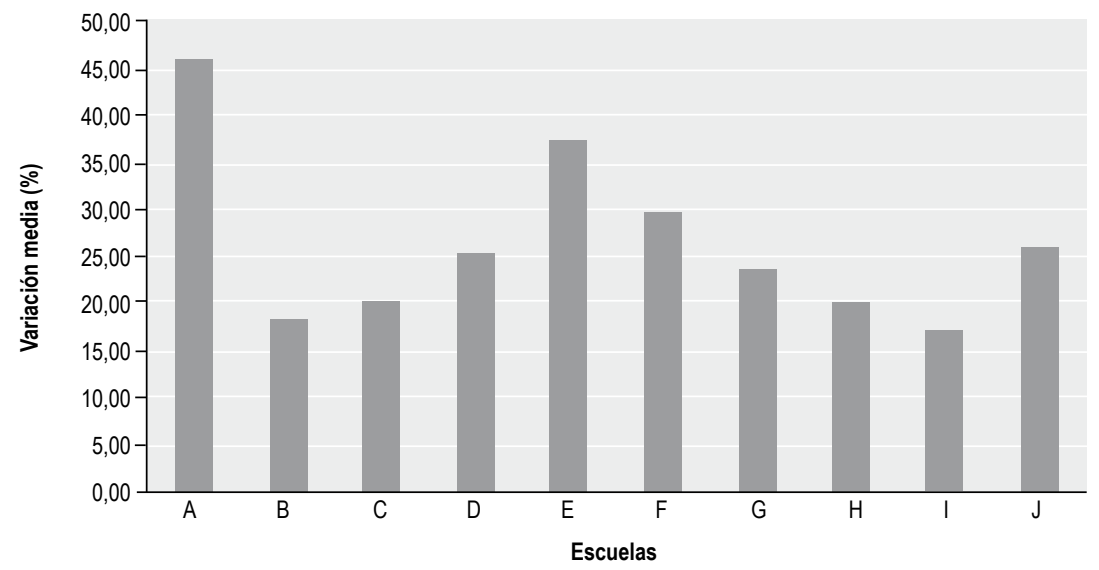

Figura 2. Escuelas prescriptivas: (A) Planificación; (B) Diseño; (C) Posicionamiento. Escuelas descriptivas: (D) Empresarial; (E) Cognoscitiva; (F) Aprendizaje; (G) De poder; (H) Cultural; (I) Ambiental; (J) De configuración.

Fuente: Elaboración propia (2010).

De los resultados presentados, se puede afirmar que en el grupo de empresas encuestadas no hay una clara tendencia o identificación con respecto de alguna de las escuelas del pensamiento estratégico, en particular, en lo referido al proceso de formulación estratégica. En efecto, no hay suficiente homogeneidad en las respuestas dadas como para poder detectar cuál escuela del pensamiento estratégico (a partir de la aplicación de sus premisas) tiene mayor presencia en alguna de las empresas. Ello dificulta aún más su interpretación de manera grupal (las cinco empresas) y limita la sectorizaron (segmentación) de los resultados por tipo de empresa, tamaño, ventas o jerarquías, entre otros posibles criterios de sectorización. Por tanto, no se puede afirmar que alguna de las escuelas del pensamiento estratégico influya de manera significativa ni en el grupo de empresas estudiadas ni en alguna de dichas empresas en especial. 
Ante ello, se procedió en primer lugar a identificar aquellas premisas con mayor promedio y menor dispersión relativa (\%) a fin de precisar las proposiciones que dan lugar al proceso de formulación estratégica. En segundo lugar se detectaron aquellas premisas con menor promedio y mayor dispersión relativa (\%) con el propósito de señalar las proposiciones con menor incidencia al momento de crear las estrategias en el grupo de empresas seleccionadas:

\section{Premisas con mayor incidencia:}

1. El proceso de formulación de estrategias se desarrolla en etapas claras, cada una de ellas delineada a través de listados y sustentada por técnicas.

2. Luego de la formulación de estrategias, se presta minuciosa atención a los objetivos, presupuestos, programas y planes operativos de diversos tipos.

3. El mercado (contexto) es económico y competitivo.

4. La creación de estrategia es un proceso de interacción social.

5. La cultura organizacional orientan la formulación de estrategias.

6. Las estrategias provienen de un proceso controlado y consciente de planificación formal.

7. Los analistas juegan un papel fundamental en este proceso.

8. El liderazgo tiene como misión leer el entorno y asegurar una adaptación correcta por parte de la organización.

9. La cultura tiende a promover cambios de posición dentro de la perspectiva estratégica general de la organización.

10. La clave de la gerencia estratégica de la organización está en reconocer la necesidad de transformación.

\section{Premisas con menor incidencia:}

1. La organización adopta una forma particular de estructura que combina con un tipo particular de contexto.
2. El proceso de formulación de estrategia está arraigado en la experiencia y la intuición del líder.

3. La formulación de estrategia es un proceso que tiene lugar en la mente del estratega.

4. Primero. las estrategias aparecen como patrones salidos del pasado; solo después aparecen como posibles planes para el futuro; y por último, como perspectivas para orientar la conducta general.

5. La formulación de estrategia es un proceso deliberado.

6. La responsabilidad por el proceso general de formulación de estrategias descansa en el directivo superior.

7. La responsabilidad por el control y el conocimiento descansa en el directivo principal.

8. Las mejores estrategias vienen de un proceso de diseño individualizado.

9. El modelo de formulación de estrategia es simple e informal.

10. Las estrategias son difíciles de obtener.

A partir de estas conclusiones se puede afirmar que las escuelas cuyas premisas tienen mayor influencia al momento de hacer la formulación estratégica son de Planificación, de Posicionamiento, Cultural, Ambiental y de Configuración.

Las escuelas cuyas premisas tienen menor influencia son Empresarial, Cognoscitiva, de Aprendizaje y de Diseño.

\section{CONCLUSIONES}

Con el propósito de analizar el proceso de formulación estratégica de un grupo de empresas del sector alimentos y bebidas del Estado Carabobo, y partiendo de los resultados presentados, se puede concluir que en el grupo de empresas encuestadas no hay una clara tendencia o identificación con respecto de alguna de las escuelas del pensamiento estratégico, en particular, en lo referido al proceso de formulación estratégica. En efecto, no se percibe suficiente homogeneidad en las 
respuestas dadas como para poder detectar qué escuela del pensamiento estratégico (a partir de la aplicación de sus premisas) tiene mayor presencia en alguna de las empresas, lo que dificulta aún más su interpretación de manera conjunta entre las 11 empresas. Por tanto, no se puede afirmar que alguna de las escuelas del pensamiento estratégico influya de manera significativa ni en el grupo de empresas estudiadas, ni en alguna de dichas empresas en especial.

Ante ello, se considera que el proceso de formulación estratégica es de carácter prismático, por lo cual se procedió en primer lugar a identificar aquellas premisas con mayor promedio y menor dispersión relativa (\%) a fin de precisar las proposiciones que dan lugar al proceso de formulación estratégica. En segundo lugar, se detectaron aquellas premisas con menor promedio y mayor dispersión relativa (\%) con el propósito de señalar las proposiciones con menor incidencia al momento de crear las estrategias en el grupo de empresas seleccionadas.

Se puede afirmar que las escuelas cuyas premisas tienen mayor influencia al momento de hacer la formulación estratégica son de Planificación, de Posicionamiento, Cultural, Ambiental y de Configuración. Inversamente, las escuelas Empresarial, Cognoscitiva, de Aprendizaje y de Diseño presentan menor incidencia en términos generales en la formulación de estrategias en el grupo de empresas del sector alimentos y bebidas carabobeño seleccionadas. Es importante reseñar que el proceso de socialización de las estrategias a los subalternos e involucrarlos en la ejecución de estas se hace a partir de la definición de los objetivos, presupuestos, programas y planes operativos vinculados a dichas estrategias por parte de cada una de las áreas funcionales de las empresas encuestadas.

Luego de analizar el proceso de formulación estratégica dentro del grupo de empresas seleccionadas del sector alimentos y bebidas del Estado Carabobo es importante destacar que, tal como se evidenció en algunas de las investigaciones reseñadas en la Introducción, tampoco se puede precisar una clara tendencia en cuanto a la formulación y pensamiento estratégico, y se observan algunas similitudes con estos trabajos reseñados.

Por último, es indispensable reflexionar sobre la necesidad de profundizar en estudios teóricos y prácticos que contribuyan a identificar las perspectivas de integración organización-entorno (pensamiento estratégico) que las organizaciones aplican hoy en día a fin de adecuarse a los cambios que actualmente caracterizan el quehacer empresarial. 


\section{Referencias}

Ansoff, H. (1965). Corporate Strategy. New York: Mc Graw-Hill.

Cámara Venezolana de la Industria de Alimentos (CAVIDEA) (2010). Situación del sector alimentos. Perspectiva I Trimestre 2010. En CAVIDEA: Estadística. [Documento en línea]. Disponible: $<$ http://www.cavidea.org/estadisticas/Perspectivas I_2010.pdf $>$ [Consulta: 2010, agosto 16]

Castillo, R., \& Morales, A. (2006). Propuesta metodológica para el análisis empírico de los cambios ocurridos en el sector agroalimentario venezolano. Revista Agroalimentaria, 23, 57-70.

Cendrós, J. (2001). Pensamiento Estratégico. Ensayos. Colección Gerencia de la Universidad Rafael Belloso Chacín. Maracaibo, Venezuela: Ediciones Gato Azul.

Chandler, A. (1962). Strategy and Structure. Chapters in the History of the American Industrial Enterprise. Massachusetts: MIT Press.

Daft, R. (2004). Administración. México, D.F.: Thomson.

Drucker, P. (1994). The Theory of the Business. Harvard Business Review, September-October. Cambridge. Disponible en $<$ http://hbr.org/1994/09/the-theory-ofthe-business/ar/1>. Recabado el 17 de enero 2010.

De Weffer, R. (2009). Formulación de estrategias en empresas de manufactura, distribución eléctrica y minería. Revista Venezolana de Gerencia, 14(46), 215-227.

García, M., \& Sabater, R. (2004). Relaciones entre estrategia y ciclo de vida de la empresa. Revista Madri $+d$. n. 20, diciembre 2003 - enero 2004. Disponible en $<$ http:// www.madrimasd.org/revista/revista20/investigacion/ proyectos1.asp >. Recabado el 20 de enero, 2010.

Giacinti, M. (2003). Pensamiento estratégico en el negocio mundial de manzanas. Revista Agroalimentaria, 17, 49-60.

Huerta, E. \& De Bourg, J. (2009). Pensamiento estratégico: el entramado epistémico en gerencia de salud pública. $C I C A G, 6(1), 117-130$.
Learned, E. P., Christensen, C. R., Andrews, K. R. \& Guth, W. D. (1965). Business Policy: Text and Cases, Homewood, IL: Irwin edition.

Márquez, A. (2005). Permisología. [Documento en línea]. Disponible en $<$ http://webarticulista.net.free.fr/amr 200522051008.html>. Recabado el 24 de abril, 2011.

Mercado, A., Córdova, K., \& Testa, P. (2007). Tendencias organizativas y tecnológicas de la industria agroalimentaria global y su manifestación en Venezuela. Revista Agroalimentaria, 24, 85-103.

Mintzberg, H. (1994). The Fall and Rise of Strategic Planning. Harvard Business Review, pp. 107-114. Disponible en $<$ http://online.worcester.edu/external/evescio/ Principles\%20of\%20Management/strategicplan. pdf $>$. Recabado el 18 de marzo, 2010.

Mintzberg, H. (1998). El proceso estratégico. México D. F.: Prentice Hall Hispanoamericana.

Mintzberg, H., Ahlstrand, B., \& Lampel, J. (1999). Safari a la estrategia. Buenos Aires, Argentina: Ediciones Granica S.A.

Morrisey, G. (1996). Pensamiento Estratégico. Construya los Cimientos de su Planeación. México: Editorial Prentice Hall Hispanoamericana.

Morrison, J. (1994). From Strategic Planning to Strategic Thinking. On the Horizon 1994, 2(3), 3-4. Disponible en $<$ http://horizon.unc.edu/projects/OTH/2-3.asp $>$. Recabado el 16 de mayo, 2010.

Newman, W. H. (1951). Administrative Action. The Techniques of Organization and Management. En Buzell, Gale \& Sultan, Engelwood Cliffs, NJ: Prentice-Hall.

Ohmae, K. (1998). La mente del estratega. México D. F.: Editorial Mc.Graw Hill.

Perozo, E. (2006). Pensamiento estratégico y gestión de talento humano en gerentes corporativos. CICAG, 4(1), 144-170. 
Piñeiro, A., Quintero, N., \& Faria, E. (2007). Pensamiento Estratégico y Eficiencia de la Gerencia Pública en las Corporaciones Locales. Negotium, 3(8), 133156.

Ramos P. A. (2009). La estrategia para empresas con sede en el Valle de Aburrá. Revista Universidad Eafit, 45(154), 53-69.

Robert, M., \& Dias, C. (2000). Estrategia pura y simple. México D. F.: Editorial Mc. Graw Hill Interamericana Editores S.A.

Ronda, G. (2004). La integración de los niveles estratégico, táctico y operativo en la dirección estratégica. Revista-Escuela de Administración de Negocios, 052, 29-57.

Rosales, M. (2001). Planeación Estratégica, Cultura y Valores. En Pensamiento Estratégico. Ensayos. Colección de Gerencia. Maracaibo, Venezuela: URBE

Rumelt, R. (1974). Strategy, Structure and Economic Performance. Cambridge: Harvard University Press.

Rumelt, R., Schendel, D., \& Teece, D. (1994). Fundamental Issues in Strategy. En R. Rumelt, D. Schendel \&
D. Teece (Eds.) Fundamental Issues in Strategy. A Research Agenda (pp. 9-47). Boston: Harvard Business School Press.

Sallenave, J. (1993). Gerencia y Planeación Estratégica. Caracas, Venezuela: Grupo Editorial Norma.

Serna-Gómez, H. (1999). Gerencia Estratégica. Planeación y Gestión-Teoría y Metodología. Bogotá, Colombia: 3R Editores.

Silvestri, K., Silvestri, C., \& Hernández, R. (2006). Pensamiento estratégico y éxito gerencial en organizaciones empresariales. Maracaibo, Venezuela: Editorial de la Universidad del Zulia (EDILUZ).

Thompson, A., \& Strickland, A. J. (2004). Administración estratégica: textos y casos. México, D.F.: Mc Graw Hill Interamericana.

Von Neumann, J., \& Morgenstern, O. (1944). Theory of Games and Economic Behavior. New Jersey: Princeton University Press.

Woods, P. (1985). Estrategias de enseñanza. En E. Rockwell (Ed.) Ser maestro: estudios sobre el trabajo docente (pp. 121-124). México D. F.: Consejo Nacional de Fomento Educativo. 